This item was submitted to Loughborough's Research Repository by the author.

Items in Figshare are protected by copyright, with all rights reserved, unless otherwise indicated.

\title{
Packing fraction of a disk assembly randomly close packed on a plane
}

PLEASE CITE THE PUBLISHED VERSION

PUBLISHER

(C) American Physical Society

LICENCE

CC BY-NC-ND 4.0

REPOSITORY RECORD

Williams, D.E.G.. 2019. "Packing Fraction of a Disk Assembly Randomly Close Packed on a Plane”. figshare. https://hdl.handle.net/2134/1725. 


\title{
Packing fraction of a disk assembly randomly close packed on a plane
}

\author{
D. E. G. Williams \\ Department of Physics, Loughborough University, Loughborough LE11 3TU, England
}

(Received 6 January 1998)

\begin{abstract}
A simple model is used to show that, in principle, random close packing of equal disks on a plane should be stable when the packing fraction is 0.813 , the average number of contacts per disk is 3.42 , and the connectivity of the simplicial net is 4 . The assembly is unstable with respect to shear stresses, which will be a consequence of compressive stresses applied to the randomly packed assembly. In practice, the packing fraction of the assembly will increase until it reaches the value associated with the triangulated simplicial net, the regularly packed disk assembly. [S1063-651X(98)07906-9]
\end{abstract}

PACS number(s): 81.05.Rm, 82.70.-y

A two-dimensional assembly of randomly close-packed (RCP) equally sized disks may be represented by a simplicial graph in which the centers of the disks neighboring, but not necessarily in contact with, each disk are joined by straight lines. Alternatively, it may be represented by the Voronoi construction in which the lines in the simplicial net are bisected perpendicularly and the bisectors form polygons surrounding each disk. Two disks are neighbors when the bisector of the line joining their centers forms a side of their Voronoi polygons. The relationship between these two representations can be used to define a critical separation of centers beyond which two disks cannot be neighbors and leads to an average separation of noncontacting disks that are neighbors. This average separation is the basis of the calculation of a packing fraction and of an average number of contacting disks in the RCP assembly.

The geometrical construction producing the simplicial net and the Voronoi polygons is shown in Fig. 1. An origin disk $O$ is shown with three of its neighbors. The simplicial net for this cluster has the form of two triangles shown in Fig. 1. The relevant part of the Voronoi net is drawn as well in Fig. 1. The angular separation of the two disks contacting $O$ (each labeled $C$ ) is $2 \theta$ and the sides of the rhomb formed by the two triangles are all of the same length $2 r$, i.e., one disk diameter. The angle subtended by one of the contacting neighbors $C$ at the center of the origin disk $O$ is $60^{\circ}$. In other words, when $2 \theta=60^{\circ}$ the two disks $C$ are in contact with each other as well as with $O$. If $2 \theta$ is more than $120^{\circ}$ the unlabeled disk, not in contact with $O$ in Fig. 1, will contact $O$ for the densest packing, i.e., the three neighbor disks will all be in contact with $O$. At the opposite extreme the unlabeled disk will not be a neighbor to $O$ when it no longer contributes a side to the Voronoi polygon surrounding $O$. It's obvious from Fig. 1 that this happens when $2 \theta=90^{\circ}$. As $2 \theta$ is reduced from $120^{\circ}$ the shorter diagonal of the simplicial rhomb becomes longer. Correspondingly, the edge of the Voronoi polygon of the origin disk facing its noncontacting neighbor becomes smaller until $2 \theta=90^{\circ}$, when it disappears. $2 \theta=90^{\circ}$ is thus the lowest limit of $2 \theta$ for which the noncontacting disk can be taken to be a neighbor of $O$.

If we assume that all values of $2 \theta$ from $90^{\circ}$ to $120^{\circ}$ are $a$ priori equally probable, then we can say that the average value of the angles $2 \theta$ for which a noncontacting disk is a neighbor of the origin disk $O$ is $105^{\circ}$. This means that in a large assembly of disks, on average, the number $n_{c}$ of disks contacting the average origin disk will be

$$
n_{c}=2 \pi / 2 \theta=360 / 105=3.42 \text {. }
$$

If the Voronoi net has a connectivity of 3 , the average Voronoi polygon must be hexagonal so that the average number $n_{n}$ of noncontacting disks is

$$
n_{n}=6-3.42=2.58
$$

The average value of the angle $2 \theta$ can be used to calculate the packing fraction of the assembly by considering one of the simplicial triangles shown in Fig. 1. The area of the disks enclosed by the triangle is simply $A(d)=\pi r^{2} / 2$ since the total angle of the triangle must be $180^{\circ}$. The area of the triangle is $A(t)=\left[2 r^{2} \sin \left(105^{\circ}\right)\right]$ and the packing fraction $\eta$ is given by the ratio of these areas as

$$
\eta=\pi /\left[4 \sin \left(105^{\circ}\right)\right]=0.813
$$

which is very close to the measured [1] values of 0.84 \pm 0.02 , an approximately calculated value [2] of 0.82 , and Berryman's [3] computer modeled value of 0.817 .

A calculation of the number $n_{s}$ of contacting disks required to provide stability in two-dimensional random close

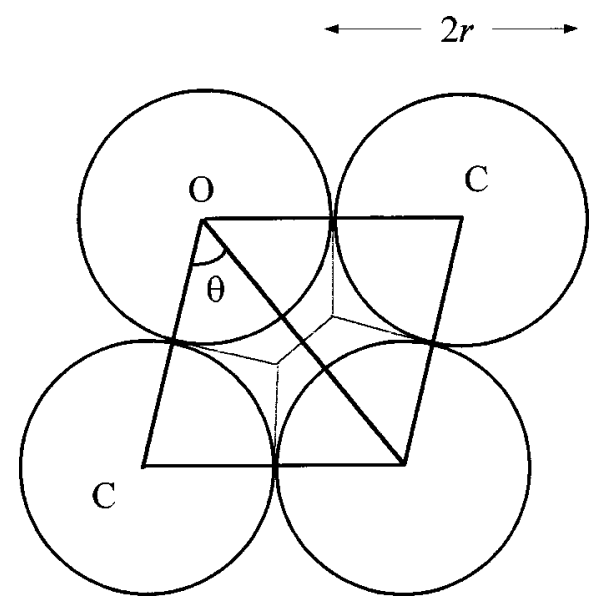

FIG. 1. Assembly of four equal disks of diameter $2 r$ packing on a plane. Lines making up the simplicial net $(-)$ and the Voronoi polygons $(-)$ are shown. 
packing [4] produces $n_{s}=3.42$, the same value as $n_{c}$ derived above. This observation seems to confirm Berryman's speculation that in two dimensions random close packing and random loose packing of equal disks occur at the same value of $\eta$, which is not the case in three-dimensional sphere packing.

This very simple derivation of the packing fraction for a RCP assembly of hard disks leads to results that are slightly different from those that have been obtained previously. Apart from its extreme simplicity, there are two reasons for its interest. The first is that it explains the apparent discrepancy between $n_{c}=3.42$ and the requirement [2] that the simplicial net should have a connectivity of 4 . The symmetry of the rhomb in Fig. 1 shows that a tiling of the plane with average rhombs would form a simplicial net with a connectivity of 4 . The second reason is that it shows that random close packing and random loose packing are the same for an assembly of equal disks. A third possible reason is that an assembly of average rhombs would be stable under a compressive stress applied along the lines of the simplicial net. A stress applied in any other direction could be represented as a combination of compressive stress along the net lines and a shear stress perpendicular to those lines. The rhombs would be unstable with respect to shear and would be distorted to form a triangulated simplicial net.

One intriguing point is that on the plane the connectivity of the disk simplicial net and the number of contacting neighbors is the same as for the curved disks on the (spherical) surface of contacts $[5,6]$ for spheres with a radius ratio of 1, i.e., for spheres packed in a face-centered-cubic structure. In both cases the vertices are 4-connected and the average number of contacts per disk is $\frac{24}{7}$. The packing fraction of the curved disks on the sphere is marginally less, at 0.802 , than that on the plane.
[1] D. Bideau and J. P. Troadec, J. Phys. C 17, L731 (1984).

[2] D. Bideau, A. Gervois, L. Oger, and J. P. Troadec, J. Phys. (Paris) 47, 1697 (1987).

[3] J. G. Berryman, Phys. Rev. A 27, 1053 (1983).
[4] W. Uhler and R. Schilling, J. Phys. C 18, L979 (1985); D. E. G. Williams, ibid. 18, L181 (1985).

[5] D. E. G. Williams, J. Chem. Phys. 87, 4207 (1987).

[6] D. E. G. Williams, Philos. Mag. B 50, 363 (1984). 